\title{
Limitantes para decodificação iterativa de sistemas MFSK multi-usuário
}

\author{
Manish Sharma e Jaime Portugheis
}

\begin{abstract}
Resumo-Neste trabalho estudamos limitantes para a detecção multiusuário conjunta iterativa utilizando grafos de fatores. Nosso canal de acesso múltiplo é um canal ruidoso com $N$ freqüiências e $T$ usuários, que pode ser utilizado para modelar um sistema FH-CDMA. O sistema é separado em duas partes: um detector multiusuário (MUD) e um código. Apresentamos um grafo de fatores para o MUD, e obtemos dele curvas de informação extrínseca, ou curvas EXIT. Combinando estas curvas com as curvas EXIT para códigos LDPC e RA, obtemos valores para a relação sinal-ruído (SNR) que limitam o desempenho do grafo. As curvas EXIT para os códigos são apresentadas pela perspectiva do detector. Os resultados são comparados com a capacidade efetiva do sistema.
\end{abstract}

Palavras-Chave-Sistema multiusuário, decodificação iterativa, grafo de fatores.

Abstract - In this work we study bounds for joint multiuser detection using factor graphs. An $N$ frequency $T$ user noisy channel is our multiple access channel, which may model a frequency hopping multiple access system. The system is separated in two parts: multiuser detector (MUD) and a code. We present a factor graph for the multiuser detector, and extract from it extrinsic information transfer (EXIT) curves. Paired with the EXIT curves of Low Density Parity Check and Repeat Accumulate codes, we obtain values for the signal to noise ratio (SNR) that bound the performance of the graph. The EXIT curves for the codes are presented from the detectors perspective. The results are compared to system's effective capacity.

Keywords-Multiuser system, iterative decoding, factor graphs.

\section{INTRODUÇÃO}

Sistemas FH-CDMA(do inglês: Frequency Hopped Code Division Multiple Access) foram introduzidos em [1] e foram propostos para sistemas de banda ultra larga (UWB - do inglês: ultrawideband) em [2]. Este sistema pode ser visto como a concatenação de um canal de acesso múltiplo com $N$ freqüências e $T$ usuários [3] com um código de espalhamento (com saltos em freqüências) para permitir o acesso múltiplo. A maioria das técnicas utilizadas para detectar as mensagens dos usuários se baseia neste código de espalhamento e num alfabeto discreto para representar a saída do canal, como em [4]-[6] por exemplo. Algumas destas técnicas também envolvem a detecção sucessiva das mensagens dos usuários, de forma que o conhecimento das mensagens já detectadas ajude a detectar as mensagens desconhecidas.

Manish Sharma e Jaime Portugheis, Departamento de Comunicações, Faculdade de Engenharia Elétrica e de Computação, Universidade Estadual de Campinas, Campinas, Brasil, E-mails: sharma@decom.fee.unicamp.br, jaime@decom.fee.unicamp.br. Este trabalho foi parcialmente financiado pela FAPESP (06/00330-7).
Neste trabalho estudamos como é o desempenho de um sistema multiusuário MFSK quando realizamos detecção/decodificação conjunta. Em [7], é mostrado que ocorre um ganho significativo quando realizamos detecção conjunta em vez de detectar a mensagem de um único usuário.

Consideramos a presença de ruídos concatenando um canal de acesso múltiplo (MAC - do inglês: Multiple Access Channel) com um canal ruidoso. A saída do canal não é discretizada. O detector multiusuário (MUD - do inglês: Multiuser Detector) é modelado como um grafo de fatores (vide [8] e as suas referências). O código de acesso com saltos em freqüências é inicialmente removido do sistema. Em vez de detectar os usuários independentemente ou seqüencialmente, todos os usuários são detectados simultaneamente e transmitem com a mesma taxa.

Dada a representação do detector como um grafo de fatores e um algoritmo de passagem de mensagens, podemos obter as curvas EXIT (do inglês: Extrinsic Information Transfer) [9],[10] para o MUD por simulação. Em vez de combinarmos o detector com parte do código como em [11],[12], combinamos as curvas EXIT do código, de forma que curvas de vários códigos possam ser comparadas com a curva do detector. Para isso, consideramos o maior valor possível de informação que um código pode retornar para o detector, dado um valor de informação a priori. Como todos os usuários transmitem com a mesma taxa, precisamos comparar a curva EXIT do MUD com somente uma curva EXIT do código. Escolhemos utilizar códigos LDPC(do inglês: Low Density Parity Check)[13] e RA(do inglês: Repeat Accumulate)[14].

Este trabalho esta organizado da seguinte forma: na seção II descrevemos o nosso modelo do canal, o MUD e o grafo de fatores equivalente; em III obtemos curvas EXIT para os códigos LDPC e RA pelo ponto de vista do detector; na seção IV obtemos valores para a SNR que limitam o desempenho do sistema; na seção V apresentamos as nossas considerações finais.

\section{Detector Multiusuário}

O MAC ruidoso considerado inclui desvanecimento Rayleigh e ruído aditivo Gaussiano branco (AWGN) com densidade unilateral $N_{0}$. Veja [15], [7] para uma descrição completa. Neste modelo, um canal é dividido em $N$ sub-canais e é utilizado simultaneamente por $T$ usuários. O símbolo escolhido pelo $j$-ésimo usuário é $m^{j}$, um número inteiro entre 0 e $N-1$. As mensagens escolhidas por todos os usuários podem ser representadas pelo vetor $\mathbf{M}=\left\{m^{1}, m^{2}, \ldots, m^{T}\right\}$. Cada 
símbolo esta associado com um sub-canal. Cada mensagem $m^{j}$ é convertida em um vetor $\mathbf{c}^{j}$ com dimensão $N$, com termos $c_{n}^{j}$ que valem 1 se $m^{j}=n$ ou zero caso contrário.

Os elementos $c_{n}^{j}$ (um chip) estão associados a sinais com energia por chip $E_{c}$. A energia por chip se relaciona com a energia por bit transmitido por $E_{c}=E_{b} \log _{2} N$, onde $E_{b}$ é a energia por bit transmitido.

$\mathrm{O}$ vetor recebido $\mathbf{R}$ com dimensão $N$ é estatisticamente descrito em [7], onde cada termo $R_{n}$ corresponde ao $n$ ésimo sub-canal. Seja $\mathbf{C}$ um vetor $N$-dimensional com termos $c_{n}=\sum_{j=1}^{T} c_{n}^{j}$ representando o número de usuários transmitindo no $n$-ésimo chip, e seja $d=N_{0} / E_{c}$. A função de densidade de probabilidade $(p d f)$ de $R_{n}$, condicionada ao número de usuários ativos no $n$-ésimo chip, é uma distribuição exponencial com parâmetro $c_{n}+d$ :

$$
p\left(R_{n} \mid c_{n}\right)=\frac{\exp -\frac{R_{n}}{c_{n}+d}}{c_{n}+d} .
$$

\section{A. Distribuição de entrada dos símbolos}

Em [7] foi afirmado que uma distribuição uniforme nos símbolos de entrada do canal para todos os usuários sempre atinge a capacidade soma do sistema. A prova se baseava no fato de que a informação mútua é uma função côncava em função da distribuição da entrada. Isto é verdade em relação à distribuição de $\mathbf{M}$, mas não necessariamente em relação à distribuição comum a todos os usuários, sendo que esta era a variável do problema. Para casos simples com $N=2$, por exemplo, a informação mútua entre $\mathbf{M}$ e $\mathbf{R}$ não é côncava para $T>2$. A complexidade do cálculo da capacidade (feita através de integração por Monte Carlo) torna proibitiva a aplicação de algoritmos de otimização para $N>3$.

O limitante superior para a informação mútua (equações 13 e 16 de [7]), por outro lado, tem cálculo bem mais simples, e fornece valores próximos aos obtidos pela integração de Monte Carlo. Podemos aplicar algoritmos de otimização para encontrarmos o seu valor máximo em função da distribuição dos símbolos de entrada do canal. Apresentamos na figura 1 a distância quadrática entre a distribuição uniforme e a distribuição que otimiza o limitante da informação mútua, para $E_{b} / N_{0}=20 d B$. Podemos ver que, para a maioria dos pontos onde $T \leq<N$, a distribuição uniforme é a distribuição que otimiza o limitante para a informação mútua. O limitante superior para a informação mútua também possui um máximo em função do número de usuários, que ocorre para uma quantidade de usuários $T_{M A X}<N$. Para $E_{b} / N_{0}=20 d B$ e $N=8,16$ e 32 , o valor de $T_{M A X}$ é respectivamente $T=6,12$ e 25 , o que reforça a escolha da distribuição uniforme para os símbolos de entrada do canal.Estas duas características sugerem que este sistema deve operar com $T<N$ e, conseqüentemente, que cada usuário utilize a distribuição uniforme nos símbolos de entrada para que se atinja a capacidade ou um valor muito próximo, que chamaremos de capacidade efetiva.

O MUD realiza maximização a posteriori, escolhendo M que maximize a densidade de probabilidade condicional $p(\mathbf{R} \mid \mathbf{M})$. Para o caso não ruidoso [3], a distribuição que atinge a capacidade é a distribuição uniforme. Para o caso ruidoso,

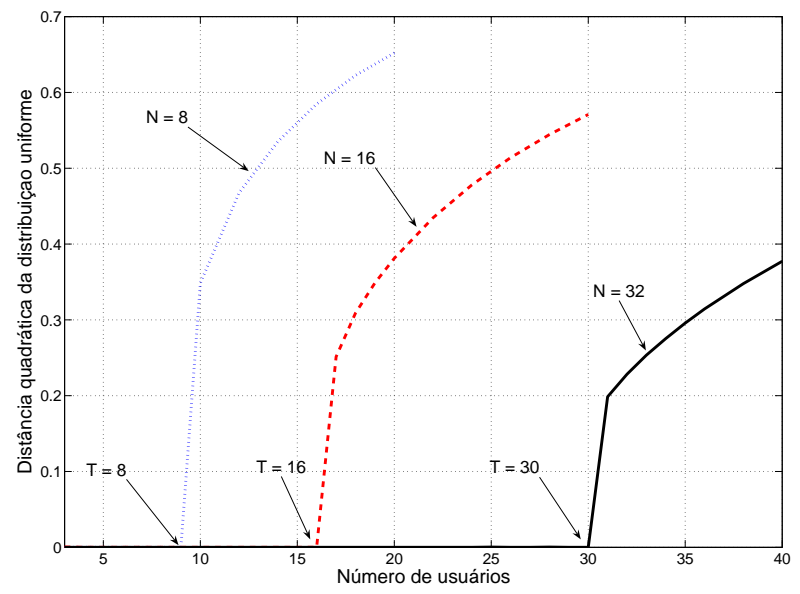

Fig. 1. Distâncias entre a distribuição que otimiza a informação mútua e a distribuição uniforme.

esta mesma distribuição maximiza o limitante superior para a capacidade quando $T<N$. Ao usarmos a distribuição uniforme, maximizar $p(\mathbf{R} \mid \mathbf{M})$ equivale a maximizar $p(\mathbf{R}, \mathbf{M})$, a densidade de probabilidade conjunta.

\section{B. Grafo de fatores correspondente}

Nos restringimos aos casos onde $N=2^{k}$, com $k$ inteiro. Desta forma, as mensagens $m^{j}$ podem ser representadas por $k$ bits $b_{0}^{j}, b_{1}^{j}, \ldots, b_{k-1}^{j}$. Como todos os sinais são ortogonais entre si, qualquer mapeamento bijetivo vai nos fornecer probabilidade de erro uniforme nos bits. Escolhemos o mapeamento natural $m^{j}=\sum_{i=0}^{k-1}\left\{b_{i}^{j}\right\} \cdot 2^{i}$. Assim, detectamos bits em vez de mensagens $N$-árias.

O conjunto de bits de todos os usuários, representado por $\overline{b_{i}^{j}}$, pode ser associado com o vetor $\mathbf{R}$ através das seguintes equações:

$$
\begin{gathered}
m^{j}=\sum_{i=0}^{k-1}\left\{b_{i}^{j}\right\} \cdot 2^{i}, \\
c_{n}^{j}=\left[m^{j}=n\right], \quad c_{n}=\sum_{i=1}^{T} c_{n}^{i},
\end{gathered}
$$

onde [.] vale 1 se o seu predicado é verdadeiro ou vale 0 se for falso. Estas equaçoes deterministicas podem ser transformadas em funções de probabilidade utilizando a mesma convenção:

$$
\begin{gathered}
P\left(c_{n} \mid \overline{c_{n}^{j}}\right)=\left[c_{n}=\sum_{j=1}^{T} c_{n}^{j}\right], \\
P\left(c_{n}^{j} \mid m^{j}\right)=\left[c_{n}^{j}=0\right]\left[m^{j} \neq n\right]+\left[c_{n}^{j}=1\right]\left[m^{j}=n\right], \\
P\left(m^{j} \mid \overline{b_{i}^{j}}\right)=\left[m^{j}=\sum_{i=0}^{k-1}\left\{b_{i}^{j}\right\} \cdot 2^{i}\right] .
\end{gathered}
$$

Juntamente com a $p d f$ de $p\left(R_{n} \mid c_{n}\right)$, podemos escrever $p\left(\mathbf{R}, \overline{b_{i}^{j}}\right)$ usando a regra da cadeia: 


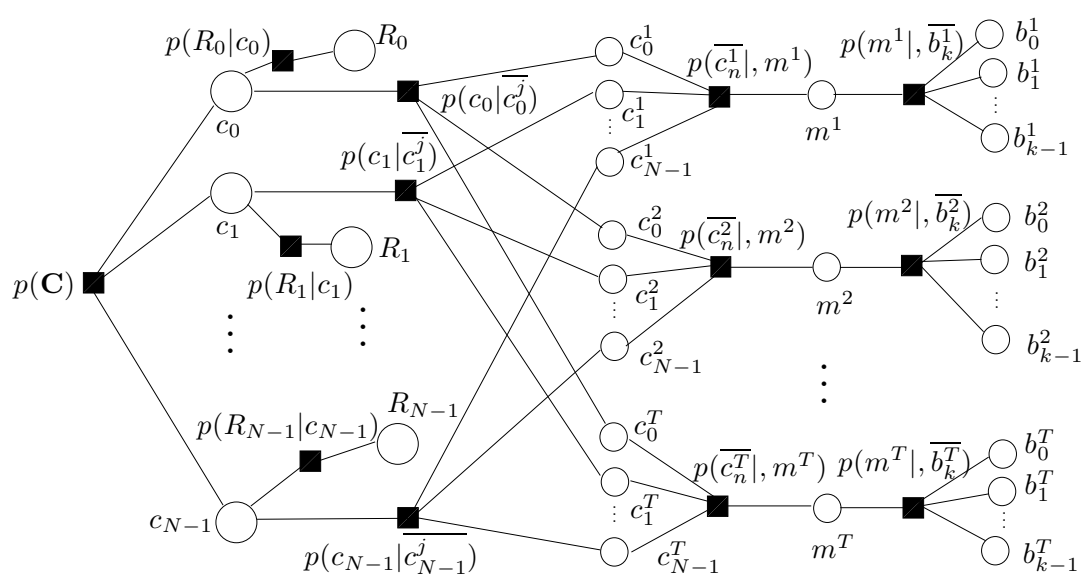

Fig. 2. Grafo de fatores de um detector para um sistema com $T$ usuários e $N$ frequiências.

$$
p\left(\mathbf{R}, \mathbf{C}, \overline{c_{n}^{j}}, \mathbf{M}, \overline{b_{i}^{j}}\right)=p(\mathbf{R} \mid \mathbf{C}) P\left(\mathbf{C} \mid \overline{c_{n}^{j}}\right) P\left(\overline{c_{n}^{j}} \mid \mathbf{M}\right) P\left(\mathbf{M} \mid \overline{b_{i}^{j}}\right) P\left(\overline{b_{i}^{j}}\right) .
$$

Os fatores (funções locais) desta equação são:

$$
\begin{gathered}
p(\mathbf{R} \mid \mathbf{C})=\prod_{n=0}^{N-1} p\left(R_{n} \mid c_{n}\right), \\
P\left(\mathbf{C} \mid \overline{c_{n}^{j}}\right)=\prod_{n=0}^{N-1} P\left(c_{n} \mid \overline{c_{n}^{j}}\right), \\
P\left(\overline{c_{n}^{j}} \mid \mathbf{M}\right)=\prod_{j=1}^{T} \prod_{n=0}^{N-1} P\left(c_{n}^{j} \mid m^{j}\right), \\
P\left(\mathbf{M} \mid \overline{b_{i}^{j}}\right)=\prod_{j=1}^{T} P\left(m^{j} \mid \overline{b_{i}^{j}}\right) .
\end{gathered}
$$

Embora tenhamos estatisticamente descrito como os bits são convertidos em valores do canal, não descrevemos todas as dependências estatísticas entre as variáveis. Como sabemos que o número de usuários é exatamente $T$, a seguinte função também é um fator do grafo:

$$
P\left(c_{0}, c_{1}, \ldots, c_{N-1}\right)=P(\mathbf{C})=\left[T=\sum_{n=0}^{N-1} c_{n}\right]
$$

A figura 2 apresenta o grafo de fatores equivalente obtido das equações 1,3-6. Este grafo nos permite computar todos os fatores locais através da troca de mensagens entre os seus nós como descrito em [8].

\section{Curvas EXIT para o detector multiusuário}

As mensagens recebidas nos nós que representam os bits são as probabilidades marginais do bit ser igual a 0 ou 1 , dada uma realização do canal. A curva EXIT é uma medida de informação mútua entre estas mensagens e o bit correspondente. Para calcular esta informação mútua, obtivemos histogramas das mensagens que chegam nos nós-bit. Para gerar estas mensagens, os valores de log-verossimilhança [16] das mensagens a priori foram modelados como em [10], utilizando a função $J(\cdot)$. Embora a saída do canal não tenha uma distribuição Gaussiana, as mensagens que passam pelo grafo são o resultado da combinação de várias variáveis aleatórias, o que gera distribuições semelhantes à distribuição Gaussiana. Observamos que as mensagens que chegavam aos nós-bit tinham este comportamento.

Um nó pode atualizar as suas mensagens de saída assim que novas mensagens de entrada estão disponíveis. Já que o grafo possui ciclos, acontece de mensagens serem passadas por um dado ramo várias vezes, e o algoritmo de passagem de mensagens não tem uma terminação natural. Considerando isto, estabelecemos um cronograma "ping-pong" de passagem de mensagens: uma vez que as mensagens são geradas nos nósbits e nós- canal $\left(R_{n}\right)$, as mensagens são atualizadas da direita para esquerda e de volta, de acordo com a figura 2. Fazer isto uma vez corresponde a uma iteração interna do MUD. Observamos que 5 iterações internas são suficientes para estabilizar a distribuição de saída das mensagens que chegam nos nós-bit. Também observamos que há uma diferença significante entre realizar uma ou 5 iterações. Para os resultados apresentados, utilizamos 20 iterações internas.

Estamos considerando um esquema de detecção paralela de mensagens, isto é, todos os usuários são detectados simultaneamente e fornecem o mesmo valor de informação $a$ priori. Como resultado, a curva EXIT do MUD é a mesma para todos os usuários, permitindo que todos transmitam com a mesma taxa.

Resultados para $N=8,16$ e 32 estão apresentados nas figuras 3 a 5, onde $I_{D}$ é a informação extrínseca fornecida pelo MUD. Para gerar as curvas escolhemos o número de usuários baseado num valor de $T_{M A X}$ para $E_{b} / N_{0}=20 \mathrm{~dB}$. Os valores de $E_{b} / N_{0}$ correspondem a energia do bit transmitido, e não à energia por bit de informação.

\section{CURVAS EXIT PARA OS CÓDIGOS DO PONTO DE VISTA DO DETECTOR}

Em [10], um código LDPC foi combinado com um detector MIMO (do inglês: Multiple Input Multiple Output). A curva EXIT do detector foi combinada com a curva do decodificador de nós-variável (VND - do inglês: Variable Node Decoder) 


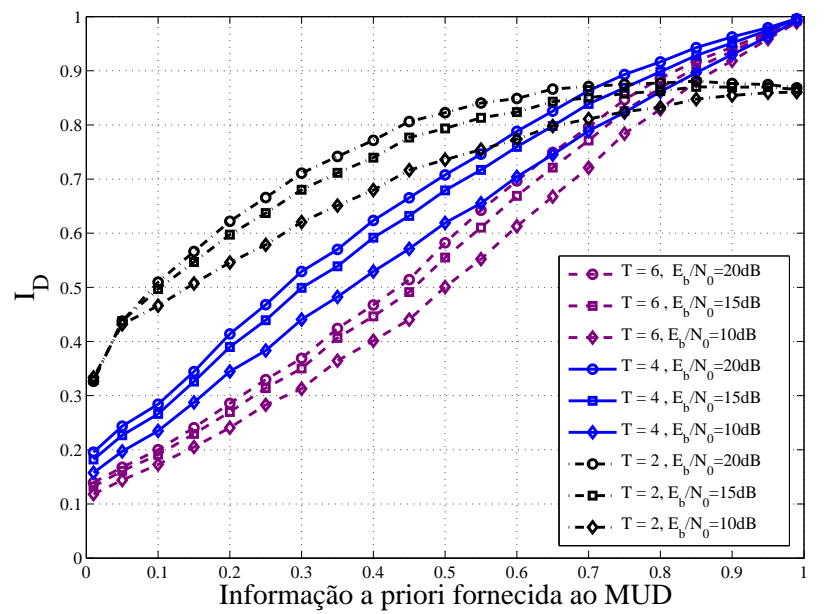

Fig. 3. Curvas EXIT para $N=8$.

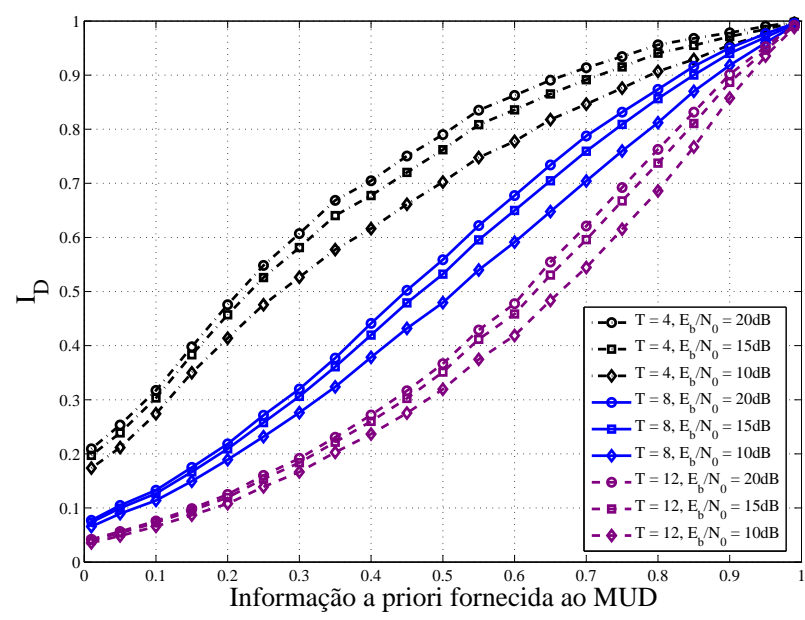

Fig. 4. Curvas EXIT para $N=16$.

para se ter uma única curva EXIT a ser comparada com a curva do decodificador de nós-paridade (CND - do inglês: Check Node Decoder). O método alternativo que propormos é combinar as curvas do VND e CND para se obter uma única curva EXIT para o código LDPC inteiro. Esta curva pode ser comparada com a curva EXIT do detector. A vantagem deste método é que, uma vez que temos a curva EXIT do detector, podemos determinar qual classe de códigos é mais apropriada para o detector em questão.

Considere a figura 6 , onde $I_{D}\left(I_{V D}\right), I_{V D}\left(I_{C V}\right), I_{C V}\left(I_{V C}\right)$ e $I_{V C}\left(I_{C V}, I_{D}\right)^{1}$ são curvas EXIT. Para combinarmos as curvas do VND e CND, consideramos que, para um dado valor de $I_{D}$ fornecido pelo detector, VND e CND vão trocar mensagens até atingir um ponto estável. Este ponto estável é

\footnotetext{
${ }^{1}$ As letras do subscrito V,C e D representam respectivamente o VND, CND e detector. A primeira letra é a origem e a segunda é o destinho. Por exemplo, $I_{V C}$ esta associado às mensagens que partem do VND em direção ao CND. Utilizamos a mesma regra para códigos RA, onde a letra A representa o acumulador.
}

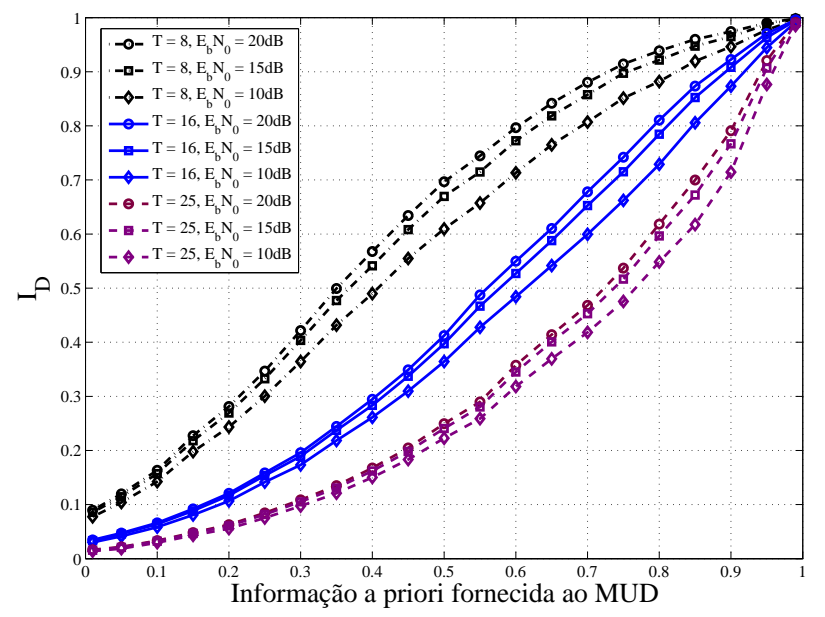

Fig. 5. Curvas EXIT para $N=32$.

o menor ponto onde as curvas $I_{V C}\left(I_{C V}, I_{D}\right)$ e $I_{C V}\left(I_{V C}\right)$ se cruzam. Isto acontece no ponto $(a, b)$ tal que $I_{V C}\left(a, I_{D}\right)=b$ e $I_{C V}(b)=a$, que pode ser numericamente obtido usando as equações de [10]. Dado este ponto, podemos obter $I_{V D}(a)$, o valor de informação a priori que o código retorna ao decetor. Ao realizarmos esta combinação obtemos uma curva para o maior valor de $I_{V D}$ que o código pode retornar, dado um valor de $I_{D}$.

Apresentamos na figura 7 curvas para alguns códigos LDPC. Os valores de $d_{v}$ (grau do VND) e $d_{c}$ (grau do CND) podem ser representados por uma seqüencia de porcentagens e valor do grau para códigos irregulares. Consideramos códigos LDPC com CND's irregulares porque eles parecem ser apropriados para a situação. Por exemplo, $\left(d_{v}=3, d_{c}=5\right.$ e $d_{v}=2, d_{c}=$ $[2 / 33,1 / 34]$ possuem a mesma taxa, e o código regular precisa de um valor menor de informação a priori para retornar 1 (convergindo para solução). Entretanto, o código irregular fornece mais informação extrínseca para valores baixos de informação a priori, exatamente onde o MUD precisa mais, como mostraremos a seguir.

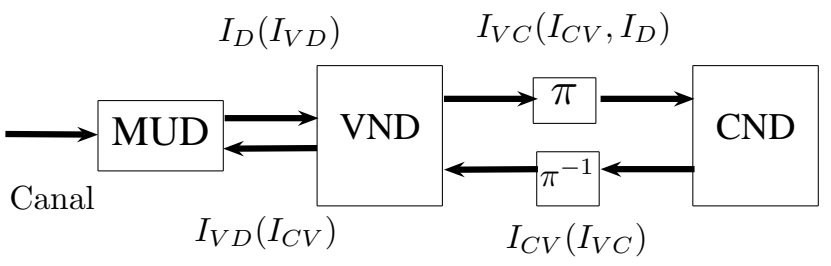

Fig. 6. Direção de troca de informações para MUD e LDPC.

\section{A. Códigos RA}

Um procedimento semelhante pode ser feito para códigos RA. Entretanto, como há três elementos (VND, CND e acumulador), o procedimento deve ser feito duas vezes para cada ponto. Considere a figura 8. Independentemente do valor de $I_{D}$, podemos obter $I_{C A}\left(I_{A C}\right)$. Depois, para um dado valor 


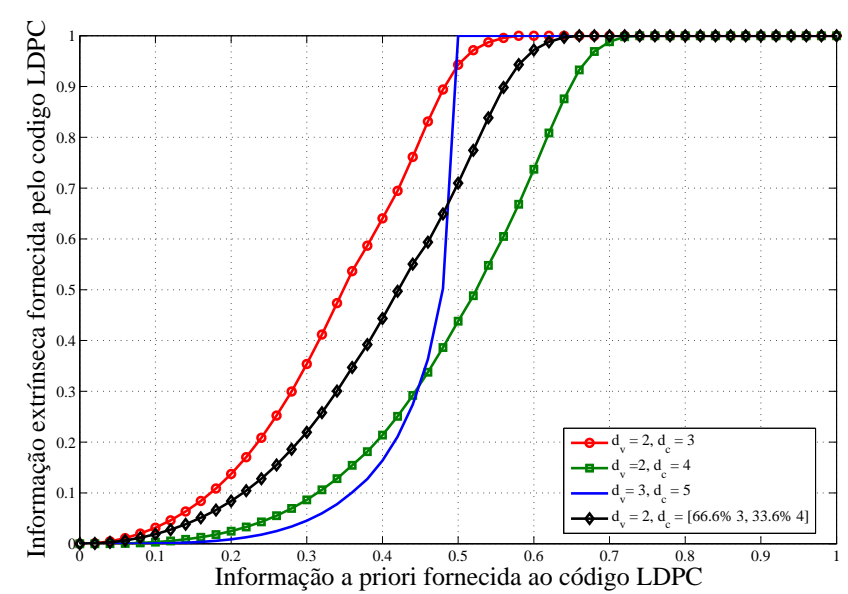

Fig. 7. Curvas EXIT para alguns códigos LDPC.

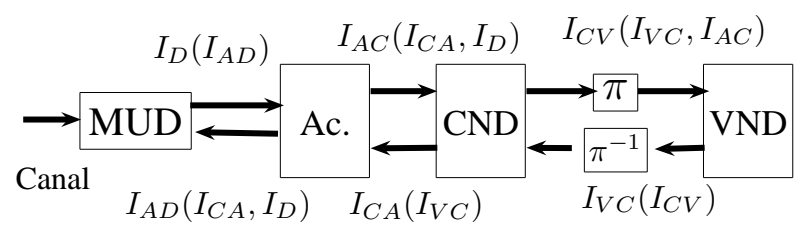

Fig. 8. Direção de troca de informações para MUD e RA.

de $I_{D}$, obtemos a intersecção de $I_{A C}\left(I_{C A}, I_{D}\right)$ e a curva combinada (fixada) de $I_{C A}\left(I_{A C}\right)$.

Em vez de usar o valor de SNR como parâmetro para a curva do acumulador, utilizamos o valor de informação a priori correspondente. Não é necessário simular o acumulador para obter a sua curva EXIT. Considere a figura 10, um subgrafo que representa a $n$-ésima entrada do acumulador. Nós temos as seguintes variáveis: $i_{n}$, o bit de entrada; $s_{m}$, o estado anterior do acumulador; $t_{n}$, o resultado da soma módulo-2 de $i_{n}$ e $s_{m}$; a saída do acumulador $o_{n}$ e o próximo estado do acumulador $s_{n}$, que são o mesmo que $t_{n}$. Seja $I_{X}^{A}$ e $I_{X}^{E}$ os valores da informação a priori e da informação extrínseca da variável $X$, respectivamente. Usando as equações de [12] obtemos o seguinte sistema:

$$
\begin{aligned}
I_{t_{n} \rightarrow}= & 1-J\left(\sqrt{J^{-1}\left(1-I_{s_{m}}^{A}\right)^{2}+J^{-1}\left(1-I_{i_{n}}^{A}\right)^{2}}\right), \\
& I_{t_{n} \leftarrow}=J\left(\sqrt{J^{-1}\left(I_{o_{n}}^{A}\right)^{2}+J^{-1}\left(I_{s_{n}}^{A}\right)^{2}}\right), \\
I_{i_{n}}^{E}= & 1-J\left(\sqrt{J^{-1}\left(1-I_{s_{m}}^{A}\right)^{2}+J^{-1}\left(1-I_{t_{n} \leftarrow}\right)^{2}}\right), \\
I_{s_{m}}^{E}= & 1-J\left(\sqrt{J^{-1}\left(1-I_{i_{n}}^{A}\right)^{2}+J^{-1}\left(1-I_{t_{n}} \leftarrow\right)^{2}}\right), \\
& I_{o_{n}}^{E}=J\left(\sqrt{J^{-1}\left(I_{t_{n} \rightarrow}\right)^{2}+J^{-1}\left(I_{s_{n}}^{A}\right)^{2}}\right), \\
& I_{s_{n}}^{E}=J\left(\sqrt{J^{-1}\left(I_{o_{n}}^{A}\right)^{2}+J^{-1}\left(I_{t_{n} \rightarrow}\right)^{2}}\right),
\end{aligned}
$$

onde a seta indica a direção das mensagens transmitidas.

Sob a hipótese de que estamos usando um acumulador grande, $I_{s_{m}}^{E}=I_{s_{n}}^{A}$ e $I_{s_{n}}^{E}=I_{s_{m}}^{A}$. Dado um valor de $I_{o_{n}}^{A}$ e $I_{i_{n}}^{A}$, podemos numericamente obter os valores de $I_{s_{n}}^{A}$ e $I_{s_{m}}^{A}$ que satisfazem esta condição, o que nos permite posteriormente calcular $I_{i_{n}}^{E}=I_{A C}\left(I_{C A}, I_{D}\right)$ e $I_{o_{n}}^{E}=I_{A D}\left(I_{C A}, I_{D}\right)$.

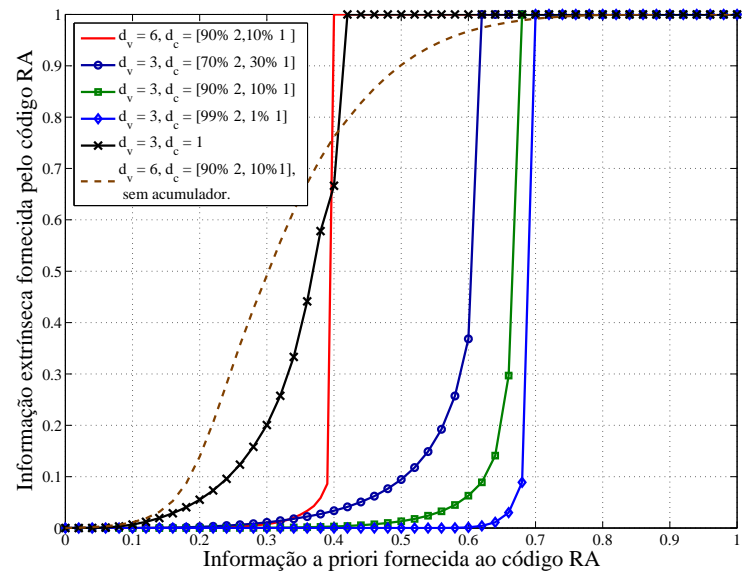

Fig. 9. Curvas EXIT para alguns códigos RA.

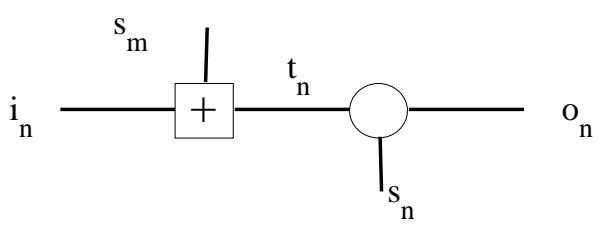

Fig. 10. Representação do acumulador.

Apresentamos as curvas para alguns códigos RA na figura 9. Como estamos usando códigos não sistemáticos, CND deve ter uma fração de nós com grau 1 ou o processo iterativo não se iniciará. Percebe-se que as curvas para os códigos RA são bem mais íngremes que as curvas dos códigos LDPC. Também apresentamos a curva de um código repetir e checar, sem acumulador, para que o efeito do acumulador possa ser notado.

\section{LIMITANTES NO DESEMPENHO DO SISTEMA}

Para alguns casos conseguimos obter códigos que permitem taxa soma $R_{S}$ próxima à capacidade efetiva $\mathcal{C}$. Estes resultados estão resumidos na tabela I. Indicamos também a capacidade efetiva para o valor de $E_{b} / N_{0}$ dado. Estes códigos foram obtidos através de tentativa e erro, escolhendo $d_{v}$ e $d_{c}$ que aproximadamente se encaixariam com a curva EXIT do MUD. Um algoritmo mais eficiente para obter os graus de distribuição forneceria códigos melhores. Algumas amostras deste procedimento podem ser vistos na figura 11 .

Podemos ver das curvas EXIT do MUD que o sistema é limitado em interferência. Para muita interferência (valor de $T$ alto), a curva EXIT do MUD começa num ponto muito baixo. Embora seja possível em alguns casos obter taxas próximas a capacidade soma efetiva usando códigos LDPC e RA, eles não parecem ser apropriados para este tipo de canal ou para detectores com curva semelhantes às curvas EXIT deste MUD. A razão para isso é que estes códigos não são muito úteis para baixos valores de informação a priori (eles retornam menos informação do que lhes foi fornecido), mas são muito 
TABELA I

COMBINAÇÕES DE SISTEMA-CÓDIGOS

\begin{tabular}{|c|c|c|c|c|c|c|c|}
\hline$N$ & $T$ & $E_{b} / N_{0}$ & Tipo & $d_{v}$ & $d_{c}$ & $R_{S}$ & $\mathcal{C}$ \\
\hline 8 & 2 & 10 & RA & 3 & $60 \% 2$ & 3.2 & 4.24 \\
& & & & & $40 \% 1$ & & \\
\hline 8 & 4 & 12 & RA & $95 \% 3$ & $70 \% 2$ & 6.04 & 6.14 \\
& & & & $5 \% 33$ & $30 \% 1$ & & \\
\hline 8 & 4 & 20 & LDPC & $67 \% 2$ & 4 & 6.66 & 7.00 \\
& & & & $33 \% 3$ & & & \\
\hline 8 & 6 & 11 & LDPC & 2 & 3 & 6 & 6.3 \\
\hline 16 & 4 & 9 & LDPC & 2 & $35 \% 3$ & 7.23 & 9.18 \\
& & & & & $65 \% 4$ & & \\
\hline
\end{tabular}

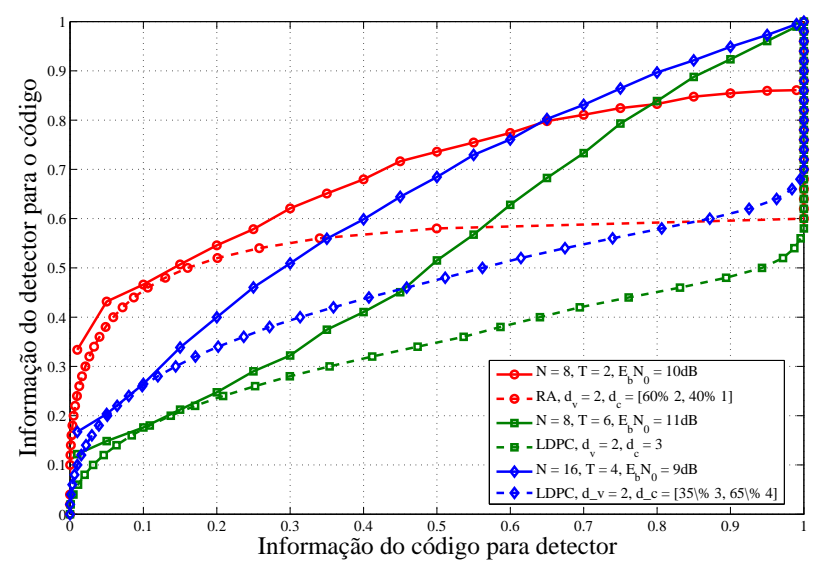

Fig. 11. Algumas combinações de curvas EXIT.

bons depois de um limiar (eles convergem para a solução sozinhos). Este comportamento não nos permite combinar apropriadamente as curvas do detector e dos códigos. Uma boa classe de códigos para este sistema seria mais balanceada, sacrificando um valor baixo de limiar por retornos melhores para baixos valores de informação a priori.

Para os códigos RA, o acumulador piora este efeito porque, para baixos valores de $I_{D}, I_{A D}<I_{C A}$ e $I_{A C}<I_{D}$. Remover o acumulador seria uma alternativa.

\section{Conclusões}

As curvas EXIT apresentadas aqui mostram que em alguns casos é possível ter um sistema de acesso múltiplo através de um canal MFSK ruidoso usando códigos LDPC e RA no lugar dos códigos de acesso. Isto fornece um sistema mais simples e com taxas mais altas. Para alguns casos, entretanto, estes códigos não são suficientes para transmissão confiável. Uma solução pode surgir ao estudarmos como outras classes de código (tais como códigos convolucionais) se comportam ao serem combinadas com este MUD. Uma vantagem deste detector é a possibilidade de que todos os usuários tenham a mesma taxa.

\section{REFERÊNCIAS}

[1] D. J Goodman e P. S Henry and V.K. Prabhu, "Frequency-hopped multilevel FSK for mobile radio", Bell Systems Technical Journal, v. 59, n. 7, pp. 1257-1275, Setembro de 1980.
[2] Lie-Liang Yang e L. Hanzo, "Residue number system assisted fast frequency-hopped synchronous ultra-wideband spread-spectrum multiple-access: a design alternative to impulse radio", IEEE Journal on Selected Areas in Communications, v. 20, n. 9, pp. 1652-1663, Dezembro de 2002.

[3] Shih-Chun Chang e J. Wolf, "On the T-user M-frequency noiseless multiple-access channel with and without intensity information", IEEE Transactions on Information Theory, v. 27, n. 1, pp. 41-48, Janeiro de 1981.

[4] U. Timor, "Improved decoding scheme for frequency-hopped multilevel fsk system", Bell Systems Technical Journal, v. 59, n. 10, pp. 1839-1855, Dezembro de 1980.

[5] T. Mabuchi, R. Khono e H. Imai, "Multiuser Detection Scheme Based on Canceling Cochannel Interference for MFSK/FH-SSMA Systems", IEEE Journal on Selected Areas in Communications, v. 12, n. 4, pp.593604, Maio de 1994.

[6] U.-C.G. Fiebig, "Iterative interference cancellation for FFH/MFSK MA systems", IEE Proceedings-Communications, v. 143, n. 6, pp. 380-388, Dezembro de 1996.

[7] M. Sharma e J. Portugheis, "Capacidade Soma para Canais de Acesso Múltiplo com T usuários e N freqüências", Anais do XXV Simpósio Brasileiro de Telecomunicações, Recife, Brasil, Setembro de 2007.

[8] F.R. Kschischang, B. J. Frey e H.-A. Loeliger, "Factor graphs and the sum-product algorithm", IEEE Transactions on Information Theory, v. 47, n. 2, pp. 498-519, Fevereiro de 2001.

[9] , S. ten Brink, "Convergence of iterative decoding", Electronic Letters, v. 35, n. 10, pp. 806-808, Maio de 1999.

[10] S. ten Brink, "Convergence behavior of iteratively decoded parallel concatenated codes", IEEE Transactions on Communications, v. 49, n. 10, pp. 1727-1737, Outubro de 2001.

[11] S. ten Brink, G. Kramer e A. Ashikhmin, "Design of low-density parity check codes for modulation and detection", IEEE Transactions on Communications, v. 52, n. 4, pp. 670-678, Abril de 2004.

[12] S. ten Brink e G. Kramer, "Design of repeat-accumulate codes for iterative detection and decoding", IEEE Transactions on Signal Processing, v. 51, n. 11, pp. 2764-2772, Novembro de 2003.

[13] R. Gallager, "Low-density parity-check codes", IEEE Transactions on Information Theory, v. 8, n. 1, pp. 21-28, Janeiro de 1962.

[14] D. Divsalar, H.Jin e R. J. McEliece, "Coding theorems for 'turbo-like' codes", Proc. 2nd. International Symposium on Turbo codes and Related Topics, pp. 1-8, Brest, France, Janeiro de 2000.

[15] O.-C. Yue, "Maximum likelihood combining for noncoherent and differentially coherent frequency-hopping multiple-access systems", IEEE Transactions on Information Theory, v. 28, n. 4, pp. 631-639, Julho de 1982.

[16] J. Hagenauer, E. Offer e L. Papke, "Iterative decoding of binary block and convolutional codes", IEEE Transactions on Information Theory, v. 42, n. 2, pp. 429-445, Março de 1996. 